\title{
ESTIMATION OF FRACTAL DIMENSIUNS FROM TRANSECT DATA
}

\author{
Craig Loehle \\ Environmental Research Division \\ Argonne National Laboratory ER/203 \\ 9700 South Cass Avenue \\ Argonne, Il 60439
}

Fractals are a useful tool for analyzing the topology of objects such as coral reefs, forest canopies, and landscapes. Transects are often studied in these contexts, and fractal dimensions computed from them. An open question is how representative a single transect is. Transects may also be used to estimate the dimensionality of a surface. Again the question of representativeness of the transect arises. These two issues are related. This note qualifies the conditions under which transect data may be considered to be representative or may be extrapolated, based on both theoretical and empirical results. 
Fractals are increasingly being used to characterize natural features such as land usage patterns, coral reef structure, rock fracture patterns, etc. (Bertuzzi et al., 1990; Bradbury et al., 1984; Burrough, 1981; Chopard et al., 1991; DeCola, 1989; Gardner et al., 1987; 1989; Krummel et al., 1987; Lam, 1990; Loehle, 1990; Milne, 1988; Palmer, 1988; Rex and Malanson, 1990; Wiens and Milne, 1989). Fractals characterize the topology of objects too irregular to be quantified as smooth Euclidean objects. For example, a straight line is a smooth Euclidean object with dimension $\mathrm{d}=1$. A coastline, however, is so irregular that its dimension is $1<\mathrm{d}<2$. In early usage, fractals were taken to be strictly nonrectifiable and self-similar. It is now recognized that self-similarity is not a necessary property, and multifractal scalings are often used.

As characterization of natural features extends to more complex objects such as surfaces, methods of measurement become more difficult. This is especially true for surfaces. Obtaining a detailed 3-D topographic description of a surface for input to a fractal calculation can be extremely time consuming and expensive. A standard statistical approach is to use a sample from a population to derive an estimate of population parameters. In the context of a surface such as a topographic surface, a transect across the surface is in a sense a sample from the entire surface. Transects have the advantage of being far cheaper to obtain than are complete spatial data because points do not need to be surveyed in, but only placed along a line by, for example, pacing along a compass bearing. Transects are also studied for their own sake, as in vegetation relevees.

When a transect is used, the dimension of the surface $\left(d_{s}\right)$ should be one greater than the dimension of a transect $\left(d_{t}\right)$ across that surface. For a flat plane $\left(d_{s}=2\right)$, a transect is just a line $\left(d_{t}=1\right)$ so that $d_{s}=d_{t}+1$. Transects have been used in a variety of disciplines. Milne (1988) used transects to characterize vegetation community structure. Palmer (1988) studied a number of actual and simulated vegetation transects. Bertuzzi et. al. (1990) used transects of soil roughness to estimate surface roughness for the quantification of rainfall effects on soil. Bradbury et al. (1984) used transects to characterize coral reef surface roughness. Burrough (1981) used transect data to estimate fractal dimensions for a variety of cases and discussed extrapolation of these values to surfaces for the evaluation of the validity of spatial interpolation in geostatistics applications. Sammis et al. (1987) extrapolated fractal dimensions of rock fracture patterns from plane sections to three dimensions. Transects are also used in oceanography and in other areas of ecology such as studies of the spatial distribution of pollutants. Not all of the above studies explicitly 
sought to obtain a fractal estimate for a surface, but it is implicit in the use of a transect that others might attempt to extrapolate the $d_{t}$ value for the transect to $a d_{s}$ value for the surface. Even if no extrapolation is done, the question arises of the representativeness of a single transect in a purely statistical sampling sense. It is not valid to consider transect sampling adequacy only in terms of sample number, because the individual samples can not be considered to be independent. Autocorrelation may occur both locally and directionally. Since a transect is a sample from a surface, the "population" of which the transect is a sample is in essence the entire surface. Thus the problem of evaluating the representativeness of a transect is structurally equivalent to the extrapolation problem.

A key assumption must be met for extrapolation of the transect dimension to the map or surface dimension: that the map is isotropic. Nonisotropis features are common in ecological and geostatistical contexts (see Isaaks and Srivastava, 1989). A nonisotropic landscape is one in which the pattern present in the landscape is different in different directions. For example, a branching pattern is not necessarily isotropic. On a nonisotropic map, a transect in one direction is often not representative. A transect down a long valley will not yield the same result as one that crosses a series of ridges and valleys.

To demonstrate this effect, an idealized artificial landscape was created consisting of a series of equal ridges (Figure 1). This map represents the kind of nonisotropic data that could be encountered in a landscape. The fractional dimension of this surface was estimated using box counting (Milne, 1988; Voss, 1988; algorithm in Loehle, submitted). Box counting quantifies the space-filling properties of objects at different scales. Changes in these properties with scale define whether the object being quantified is a solid object $(d=3)$, a linear object $(d=1)$, a flat object $(d=2)$ or something intermediate. For example, a. series of balls in a line are of dimension $d=3$ at fine scales, but at coarser scales the linear feature of the line of balls dominates, yielding $d \approx 1$. Note that in this approach $d$ is not assumed to be the same at all scales (i.e., self-similar), and $d$ may even be integer-valued. A series of $d$ values is obtained at different scales, giving a fractional dimensional profile (as in Figure 2). This multiscale approach is similar in spirit to that of Halsey et al. (1986), except that their approach is more suited to complex geometric objects such as cantor sets and chaotic attractor sets where box counting becomes inadequate. For characterizing the topology of natural surfaces, a fractional dimensional profile is probably adequate. 
As measured by box counting, the surface in Figure 1 is smooth and close to 3-D at fine resolution (open squares in Figure 2). At intermediate scales, the map approaches a 1-D topology because of the long ridges. At very coarse scales, the regularly spaced ridges give the appearance of a spatially uniform, 2-D surface. Overall, this landscape gives a Vshaped fractional dimension signature.

A one-pixel-wide slice of the surface in Figure 1 was cut out perpendicular to the ridge axes, and its dimension $\mathrm{d}_{\mathrm{t}}$ estimated by box counting. The value $\mathrm{d}_{\mathrm{t}}+1$ should equal the surface dimension at various scales by extrapolation. The results, however, show this not to be the case (Figure 2). The transect estimate is very close to the true values at very fine scales, but it deviates significantly at intermediate scales. For the five largest box sizes in Figure 2, the estimates of the two methods coincide exactly. However, this is purely an artifact of the fact that the ridges are lengthwise symmetric, perfectly straight, and regularly spaced. A series of undulating ridges would not yield the same results for the two methods at these scales.

The performance of the transect method (again, perpendicular to the ridges) is considerably worse when only one ridge is on the map (Figure 3). Crossing a single ridge with a transect is very likely in the field, where single stream valleys occur at the scale of field sampling. In this case, the full surface estimate of $d$ begins at close to 3 and falls to 1 , because at small scales the ridge is a slightly rough 3-D surface, whereas at large scales, it is viewed as a line. The transect estimate, in contrast, is highly erratic. It also appears to converge to 1 at large scales because it views the single slice through the ridge as a point $\left(d_{t}=0\right.$ so $\left.d_{s}=1\right)$ at these large scales. If the "ridge" meandered like a river course, the true $d$ would be greater than one but the transect estimate at large scales would still be $d_{t}+1=1$. Thus, the convergence of the transect estimate to the true value here is again an artifact of the perfect straightness of the ridge.

A more realistic evaluation of transect extrapolation was conducted using the spearfish data set from GRASS. GRASS is the GIS package from the U.S. Army Corps of Engineers Construction Engineering Research Laboratory. The spearfish data set covers two topographic 1:24,000 quads (Spearfish and Deadwood North) in western South Dakota (Figure 4). It includes most of the Black Hills National Forest. The fractional dimensional profile for the entire map was calculated as before. Four perpendicular transects (two $x$ and two y) were sampled across the center of the map and across the left edge (50 units in). 
The dimensionality of these two transects +1 is plotted versus the whole-map dimensionality in Figure 5. Considerable deviation is evident in the dimensional profiles extrapolated from the transects versus the estimate from the entire data set. This topography has both fine-scale and coarse-scale features. At the coarsest resolution (the largest boxes), $d$ falls below 2 because the map is rectangular. All along the profile the deviation of the transect estimates from the true value is unacceptable. This deviation results from the nonisotropic character of this map. We may also look at this result from the viewpoint of the representativeness of a single transect rather than as an extrapolation problem. The $d_{\mathfrak{t}}$ values for the four transects in Figure 5 range, over the middle spatial scales, from near 1 (a smooth line) to near 1.75 (a very irregular line) corresponding to values between 2 and 2.75 for $d_{s}$. This wide range of values indicates that single samples (transects) may not be representative. Even for an isotropic, self-similar surface, a single transect is likely to yield unreliable dimensional profiles merely because it is in essence a very small sample from a population. From the viewpoint of sampling theory, we might expect that the mean of a large number of samples would converge to the mean fractional dimensional profile. From this perspective, we can address the issue of the sample size (number and length of transects) necessary to achieve a given level of accuracy in the $d_{s}$ profile. However, one must first specify where along the profile we are most interested in accuracy (at all scales equally? smallest scales only?) and what level of accuracy is acceptable for our stated purposes. The state of the art does not yet allow us to define these parameters because we do not have a sampling theory framework within which we can determine statistical power and consequent sample sizes. Further studies would be needed to investigate these issues.

A further difficulty arises when the data being measured along a transect differ from an elevation. For example, one may measure a pollutant concentration along a transect. To use box counting, the $\mathrm{z}$ metric (here a concentration) must be in the same units as the $\mathrm{x}-\mathrm{y}$ metric (distance). The $\mathrm{z}$ data could be rescaled according to Loehle (submitted), but doing so requires data on the distribution of the variance of $z$ across the map. If these more complete data were available, it is not clear why one would be using transect data. Thus, for transect data where the $\mathrm{z}$ metric is not in elevation units, one can not extrapolate to the plane at all, but must fall back on methods such as the linear (transect) semivariogram (Isaaks and Srivastava, 1989). 
Thus we may summarize the necessary conditions for extrapolation of transect estimates of fractal dimensions as: (1) the values measured along the transect must be in units of distance (length) and, (2) the system must be isotropic.

To ensure that the surface is isotropic, the following procedure is necessary unless corrollary data are available. At least two perpendicular transects should be measured. If both transects produce a single straight line on the $\log$-log plot of boxes filled versus box size, as evidence of self-similarity (or if the lines on plots of the type used here are horizontal), and if $d$ is the same for both transects as evidence of isotropic patterning, then the transect data may validly be extrapolated to estimate a single surface dimension $d$. The two conditions of self-similarity and isotropism are both necessary and together are sufficient for extrapolation of transect data to estimate a single value for whole-surface dimensionality. Only in this situation is it meaningful to talk about "the" fractal dimension of a surface. If a surface is not self-similar, then its shape (degree of linearity, roughness, etc.) changes with scale, as in the example used here (Figure 2, V-shaped profile). A nonfractal object may have a multifractal surface (one in which different degrees of roughness or complexity are evident or operate at different scales) or a nonfractionally dimensioned (i.e., smooth) surface. Transect data may be extrapolated to find the multifractal profile (as in Figure 2) if and only if the pcrpendicular transects give the same fractional dimensional profile shape, indicating isotropisrn. However, in multifractal cases the representativeness of a single transect is much less than in the self-similar case, and the adequacy of even two perpendicular transects becomes doubtful. In this case, a sufficient condition would be to use multiple transects, with the number being determined by convergence of the mean profile to a constant shape. If too many transects are required to achieve convergence, it may turn out to be better to survey the entire surface.

Overall, then, it is insufficient to treat transect shape data in a statistical vacuum. The representativeness of a transect must be evaluated in the context of necessary conditions for extrapolation and sufficiency in terms of adequate number of transects for statistical reliability.

\section{ACKNOWLEDGEMENTS}

This paper was prepared in connection with work done under contract W-31-109-ENG-38 with the U.S. Department of Energy, Office of Energy Research, Office of Health and 
Environmental Research. Helpful reviews were provided by Barry Lesht and Carol Rosignolo. Programming assistance was provided by Carol Rosignolo. Topographic data provided by Marc Levine. Remote sensing data were collected and analyzed under contract DEAC-0976SR00-819 with the U.S. Department of Energy. 


\section{LITERATURE CITED}

Bertuzzi, P., G. Rauws, and D. Couralt. 1990. Testing roughness indices to estimate soil surface roughness changes due to simulated rainfall. Soil and Tillage Res. 17:87-89.

Bradbury, R. H., R. E. Reichelt, and D. G. Green. 1984. Fractals in ecology: methods and interpretation. Marine Ecology Progress Series 14:295-296.

Burrough, P. A. 1981. Fractal dimensions of landscapes and other environmental data. Nature 294:240-242.

Chopard, B., H. J. Herrmann, and T. Vicsek. 1991. Structure and growth mechanism of mineral dendrites. Nature 353:409-412.

DeCola L. 1989. Fractal analysis of a classified landsat scene. Photogrammetric Engineering and Remote Sensing 55:601-610.

Gardner, R. H., B. T. Milne, M. G. Turner, and R. V. O'Neill. 1987. Neutral models for the analysis of broad-scale landscape pattern. Landscape Ecology 1:19-28.

Gardner, R. H., R. V. O'Neill, M. G. Turner, and V. H. Dale. 1989. Quantifying scaledependent effects of animal movement with simple percolation models. Landscape Ecology 3:217-227.

Halsey, T.C., M. H. Jensen, L.P. Kadanoff, I. Procaccia, and B. I. Shraiman. 1986. Fractal measures and their singularities: the characterization of strange sets. Phys. Rev. A 33:1141-1151.

Isaaks, E. H., and R. M. Srivastava. 1989. Applied Geostatistics. Oxford University Press, NY.

Krummel, J. R., R. H. Gardner, G. Sugihara, R. V. O'Neill, and P. R. Coleman. 1987. Landscape patterns in a disturbed environment. Oikos 48:321-324. 
Lam, N. S.-N. 1990. Description and measurement of Landsat TM images using fractals. Photogrammetric Engineering and Remote Sensing 56:187-195.

Loehle, C. 1990. Home range: A fractal approach. Landscape Ecology 5:39-52.

Loehle, C. Submitted. Rescaled box-counting for the topological analysis of spatial data. To: Landscape Ecology.

Milne, B. T. 1988. Measuring the fractal geometry of landscapes. Applied Mathematics and Computation 27:67-79.

Palmer, M. W. 1988. Fractal geometry: a tool for describing spatial patterns of plant communities. Vegetatio 75:91-102.

Rex, K. D. and G. P. Malanson. 1990. The fractal shape of riparian forest patches. Landscape Ecology 4:249-258.

Sammis, C. G., G. King, and R. Biegel. 1987. The kinematics of gouge deformation. Pure and Applied Geophysics 125:777-812.

Voss. R. F., 1988. Fractals in nature: from characterization to simulation. pp. 21-70 in: H.O. Peitgen and D. Saupe (eds.). The Science of Fractal Images. Springer, N.Y.

Wiens, J. A. and B. T. Milne. 1989. Scaling of "landscapes" in landscape ecology, or landscape ecology from a beetle's perspective. Landscape Ecology 3:87-96. 
Figure 1. Artificial landscape of regular repeating ridges. There is some vertical exaggeration of the ridges so that details of their shapes can be seen. 


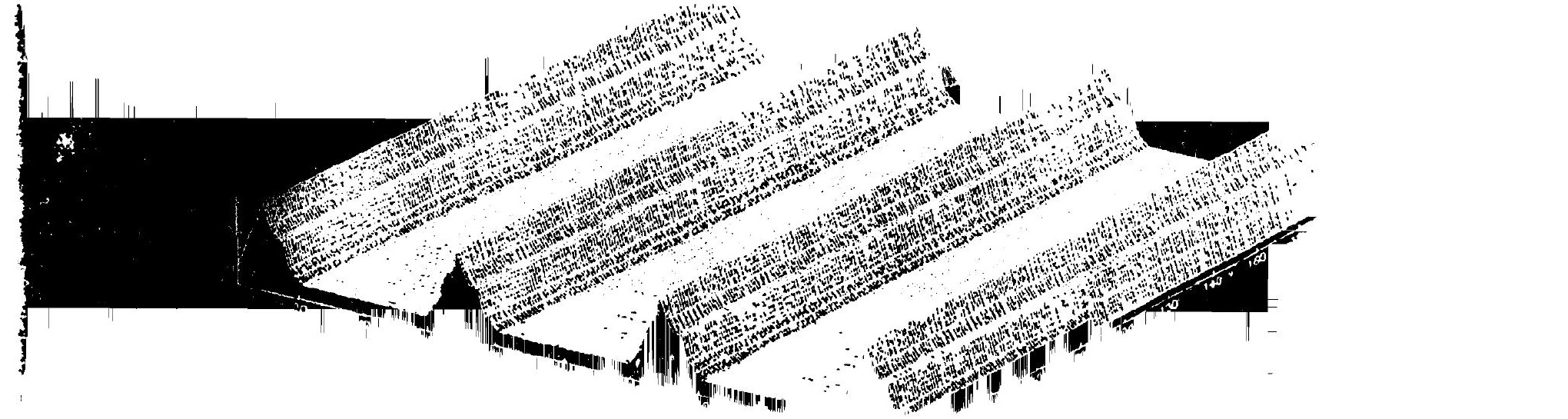


Figure 2. Fractional dimensions estimated at several length scales for the surface and by a perpendicular transect for landscape in Figure 1. 


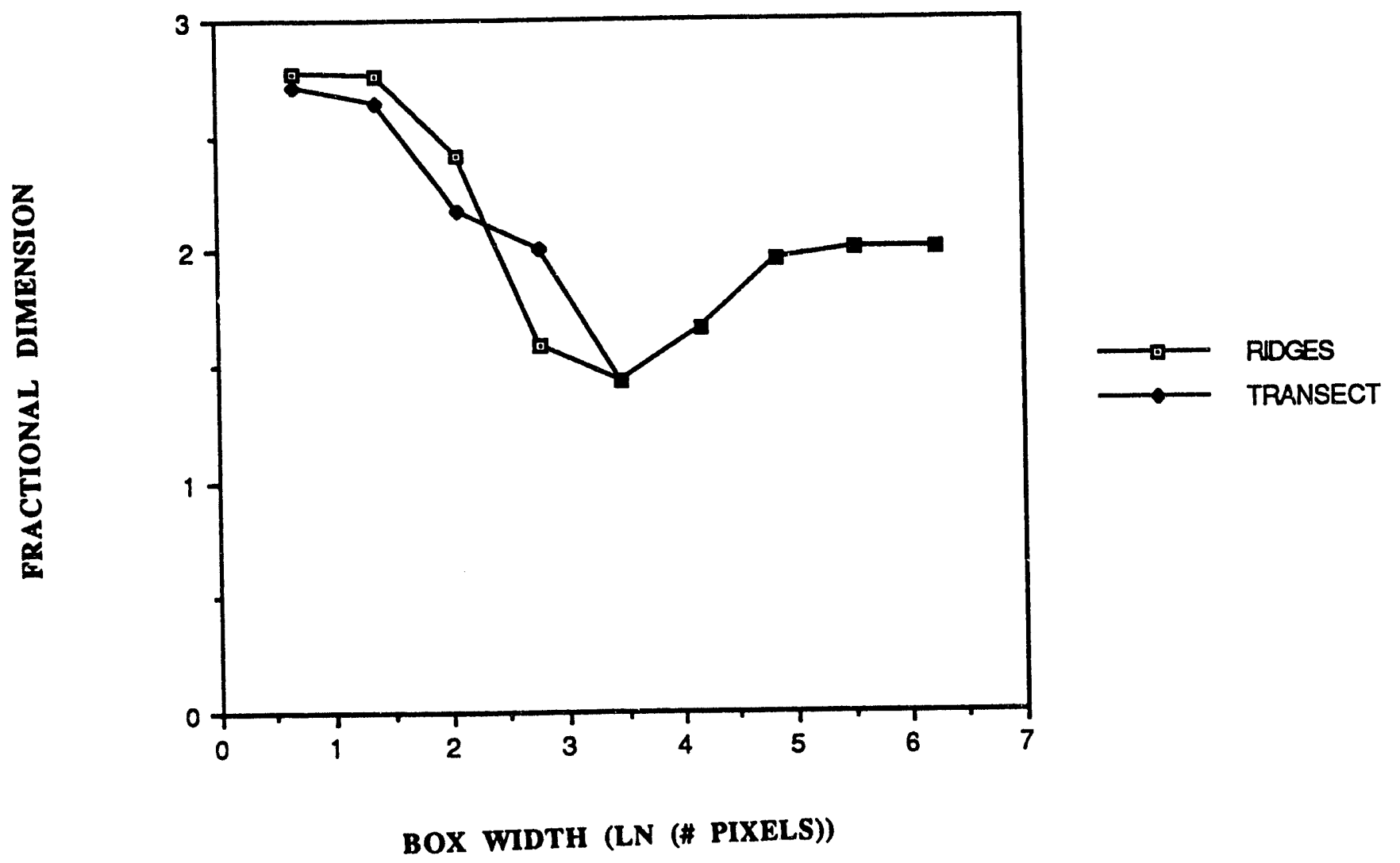


Figure 3. Fractional dimensions estimated at several scales for the surface and by a perpendicular transect for a single ridge from Figure 1 . 
FRACTIONAL DIMENSION




Figure 4. Spearfish topographic data set from GRASS (see text). 


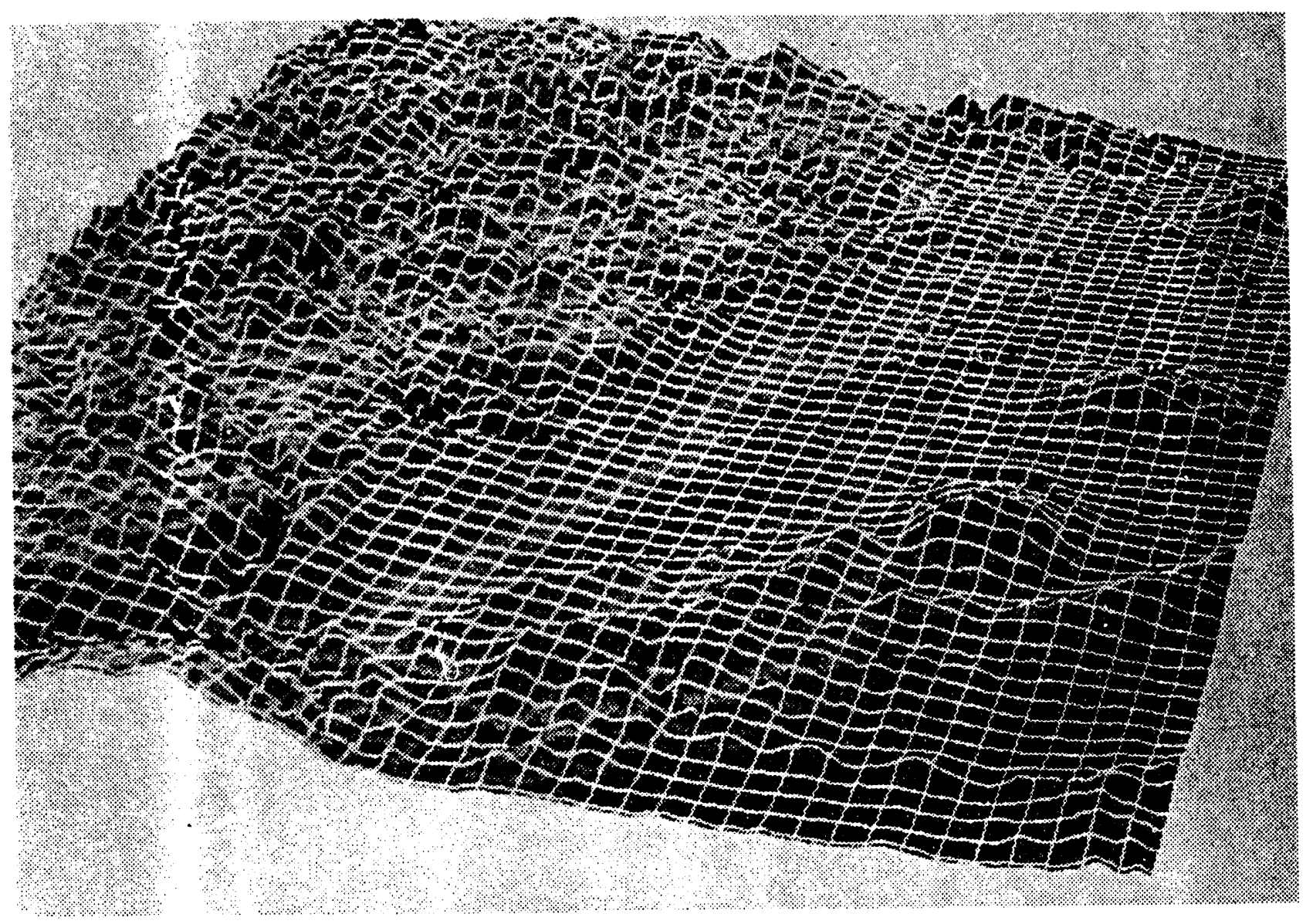


Figure 5. Fractional dimensions vs scale for the spearfish topography and as estimated by four perpendicular transects across the map. 


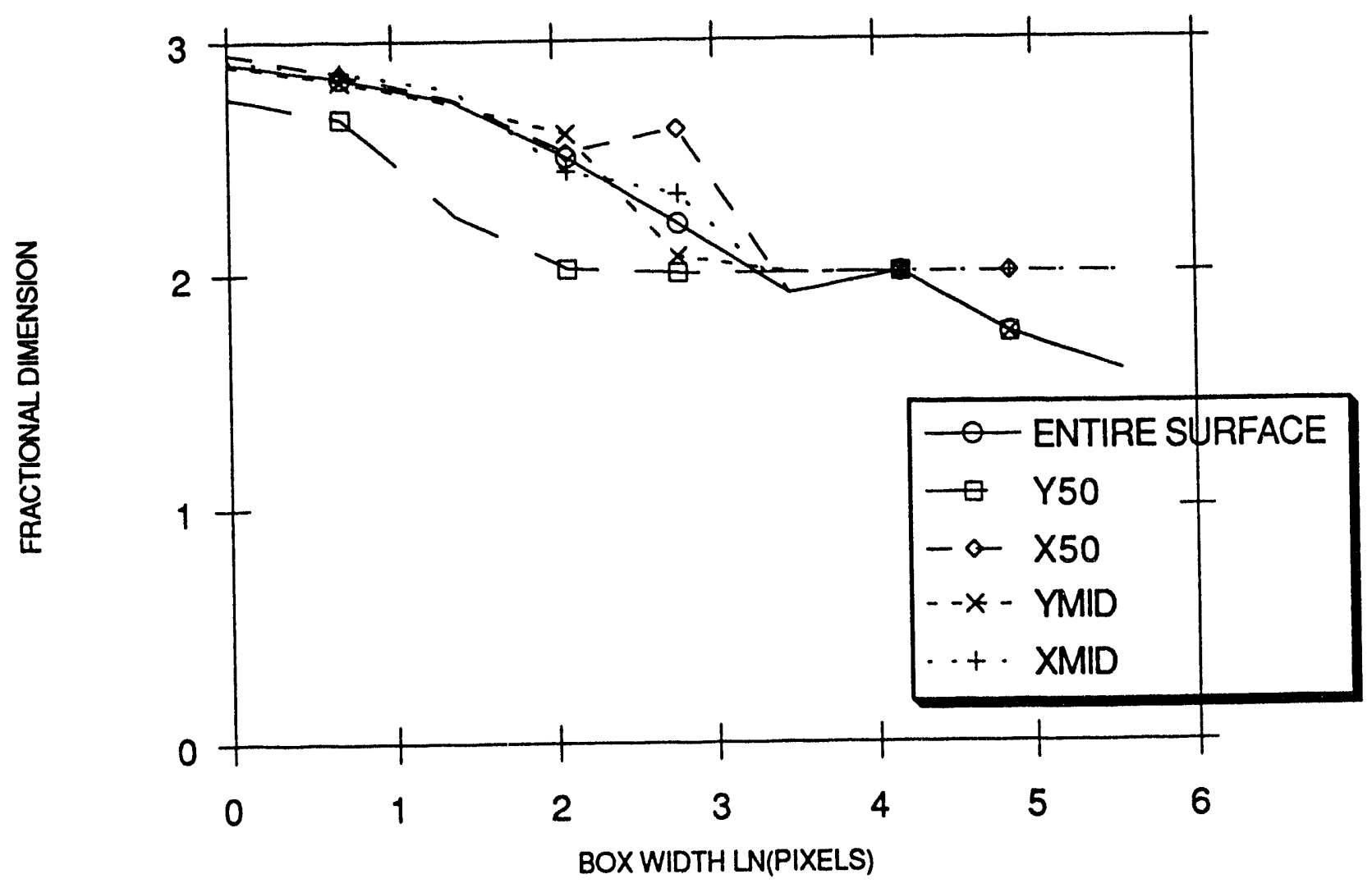



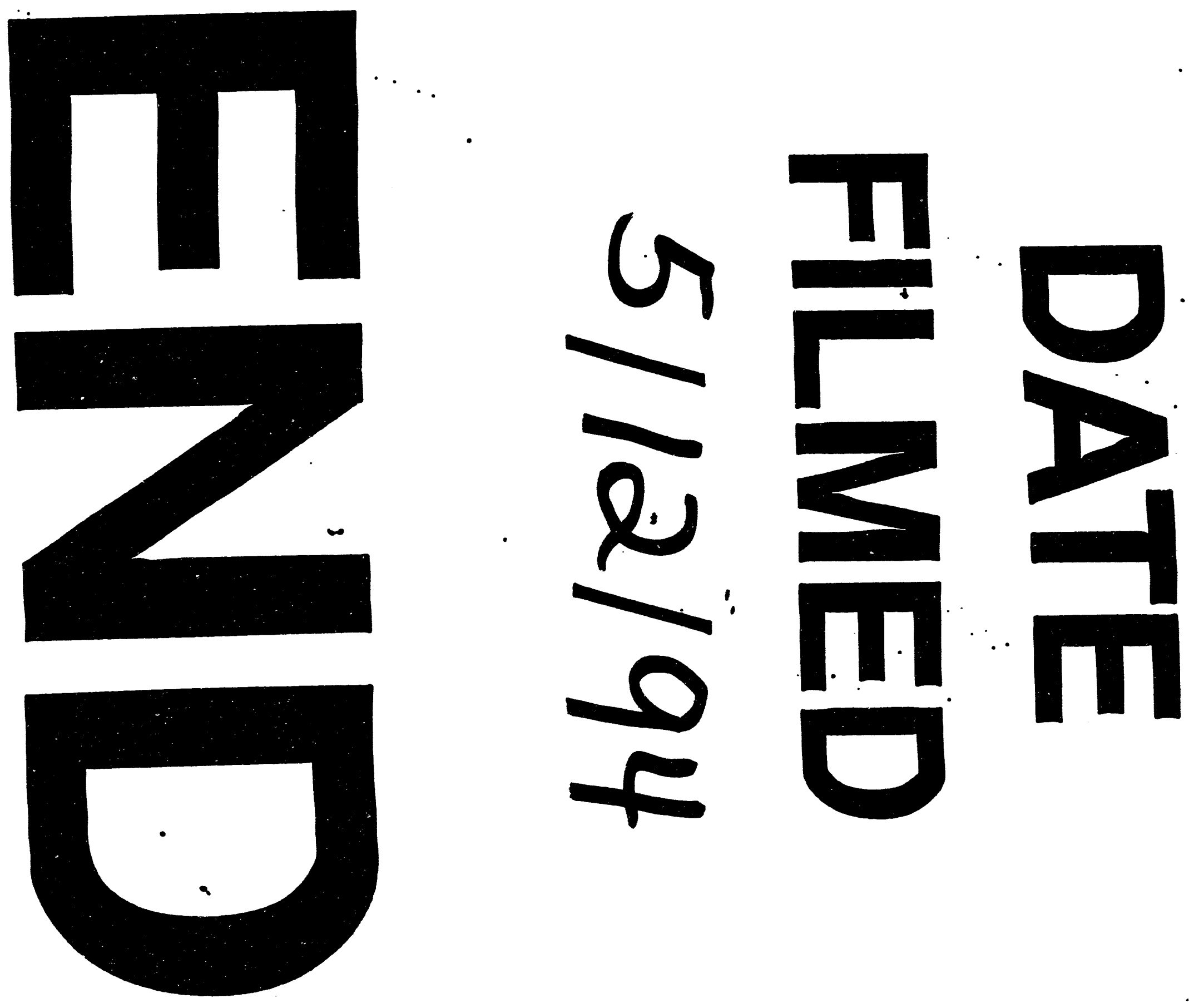
